\title{
Quand l'Histoire se fond dans un paysage remémoré : l'écriture de la mémoire et de l'oubli dans Sarah, feuille morte de Jean-Claude Pirotte
}

\author{
When History melt into the recalled landscape: \\ the writing of memory and oblivion in Sarah, feuille morte \\ by Jean-Claude Pirotte
}

\author{
Alicja Ślusarska \\ Université Marie Curie-Skłodowska, Lublin \\ e-mail: alicja.slusarska@poczta.umcs.lublin.pl
}

\begin{abstract}
The purpose of this article is to analyse the primordial role of the memory - and his counterpart, the forgetting - in the construction of identity landscape by Belgian writer Jean-Claude Pirotte. Focusing on the memory's landscapes in Pirotte's novel, Sarah, feuille morte, we show in what ways the personal and collective memory (Belgian as well as Lotharingian) combine and what are the terms that allow a singular recollection to join or to expand the shared memory.
\end{abstract}

Keywords: personal and collective memory, oblivion, identity, Belgian novel

En attendant Ulysse, sa femme Pénélope déclare aux prétendants qu'elle ne peut contracter un nouveau mariage avant qu'elle achève une toile destinée à envelopper le corps de son beau-père qui va mourir. Ainsi, pendant vingt ans, Pénélope allègue cet ingénieux prétexte, sans que sa tapisserie s'achève jamais, car elle défait la nuit ce qu'elle avait fait le jour. Jean-Claude Pirotte, écrivain et aquarelliste belge, dont l'œuvre se refuse à obéir au principe de non-contradiction, compare, à son tour, la vie humaine à la toile de l'épouse d'Ulysse, en écrivant : «Quoi de plus désolé que les temps ordinaires, qui tissent une existence en l'annulant : l'ouvrage de Péné- 
lope » (Pirotte, 1991 : 27). Le roman pirottien, Sarah, feuille morte, publié en 1989, en est la meilleure preuve.

La narratrice et protagoniste Sarah, une jeune femme de 20 ans, qui cherche à reconstituer son passé en remplissant les pages de son cahier, se perd dans le labyrinthe des paysages mémoriels. Ceux-ci dévoilent le caractère sélectif, partiel et souvent biaisé de la mémoire, ainsi que la difficulté de réconcilier des mémoires hétérogènes et antagonistes dans une vision unitaire. Sa tentative de l'écriture suggère que, derrière toute création originale, il y a une part d'oubli ou, tout au moins, une relation avec le temps qui ôte toute pertinence à la distinction entre mémoire et oubli. Ainsi, les visages du passé, auxquels revient Sarah, « se superposent et se confondent. Ils s'annulent » (Pirotte, 1989 : 34) - comme s'ils étaient cousus par le fil de Pénélope. Or, l'impuissance maladive de Sarah d'affronter le présent, dans lequel les autres la croient vivante, bien qu'elle se voie comme une " feuille morte " ou une « noyée », renforce son désir de "flâner en elle-même sans méthode et sans but » (Pirotte, 1989 : 17) - ce que l'écriture de la mémoire participant de celle de l'oubli rend possible.

L'observation du temps est avant tout l'instrument d'une enquête sur soi. Le retour vers le passé est d'autant plus difficile que Sarah semble être dépourvue de repères identitaires fondamentaux ; elle écrit : «Je n'ai pas de souvenirs. Je n'ai pas de langue natale. Le français, je l'ai appris dans les livres » (Pirotte, 1989: 10). Sa petite enfance, marquée par une suite de déménagements - de la Caroline du Nord au Québec, du Danemark à l'Autriche - éveille chez l'héroïne un sentiment de dépaysement. En outre, après la séparation de ses parents, lorsque son père dépose Sarah adolescente à Reims pour la mettre sous la tutelle de ses tantes, la narratrice se considère comme une orpheline volontaire, en disant : «Je n'ai pas de père, je n'aurai jamais de père » (Pirotte, 1989 : 34). On dit que notre vraie tombe est la mémoire des vivants et nous sommes véritablement morts, quand ils nous oublient. C'est pourquoi, l'abandon de la part de son géniteur est intimement lié pour Sarah à l'oubli, donc à sa propre mort symbolique ; Sarah note : "Toutes ces années sans mon père, c'était la mort déjà. La mort, je n'ai donc que ce mot galvaudé à la bouche » (Pirotte, 1989: 33). Et c'est justement le message sur le décès du père qui déclenche l'écriture de Sarah et lui donne un sens. Car «c'est aux morts que l'on s'adresse avec le plus d'aisance. Les liens de l'absence, au moins, ne se relâchent pas. Ce serait avec mon père qu'inconsciemment je me serais mise à converser » (Pirotte, 1989 : 31). Paradoxalement, la mort du père, la figure dont l'existence a été marquée par l'ignorance et le silence vis-à-vis de sa fille, devient donc pour Sarah la seule possibilité de dialoguer avec lui.

La descente volontaire de la narratrice dans le monde des morts l'associe à une autre figure mythique, celle d'Orphée. Sarah compare même les courants de la mémoire à un affluent de l'Achéron (Pirotte, 1989 : 62). Celui-ci, dans la mythologie grecque, désigne une branche de la rivière souterraine du Styx, sur laquelle Cha- 
ron transportait en barque les âmes des défunts vers les Enfers. À l'instar d'Orphée, qui veut libérer sa bien-aimée Eurydice du royaume des Ombres, Sarah « cherche partout sans la reconnaître la petite fille qui aimait le ciel » (Pirotte, 1989 : 62). Sa quête de l'enfance perdue s'avère impossible comme d'ailleurs celle d'Orphée, car « la source était égarée, une clé liquide égarée » (Pirotte, 1989 : 63). Tous deux déplorent donc que les fleuves ne remontent pas leur cours.

Dans le film de Jean Cocteau, Orphée ${ }^{1}$ glisse dans un autre monde par le biais du miroir. Chez Pirotte, le motif du miroir est récurrent. Il résulte d'une quête constante de l'identité que Sarah n'arrive pas à assumer : «Je me concède un rien de fausse vie, comme tout le monde. À moi, devant ma glace, je m'offre telle que je suis. Et je me chasse de ma vue » (Pirotte, 1989: 122), dit-elle. En se souvenant de sa rencontre avec son père à Vienne, Sarah avoue qu'elle «n'a gardé que des rares images, des reflets de miroirs, qui cachent la profondeur dans la surface » (Pirotte, 1989 : 32). Le miroir chez Pirotte, à l'instar du travail de la mémoire et de son pendant, l'oubli, fonctionne donc simultanément comme une représentation de la vérité et comme son inverseur.

Tout souvenir est entaché d'imprécision, d'effacement, de lacune, c'est-à-dire de formes d'oubli ${ }^{2}$. Face à cette amnésie partielle, le passé perdu ne survit que grâce à la contribution substitutive de l'imaginaire. Sarah avoue qu'elle invente, elle joue donc à cache-cache avec elle-même autant qu'avec le lecteur. Ainsi, au fil des pages, la narratrice compare les personnages de son passé flou aux marionnettes, auxquelles elle accorde les noms fabuleux, soit du marquis Carabas de Perrault, soit de Pierrot dans la Commedia dell'Arte. En s'inspirant des contes d'enfance, Sarah recourt à l'image d'un chat qui l'examine attentivement et lui pose des questions. En effet, dans ses écrits, l'anthropomorphisation des animaux va de pair avec l'animalisation des hommes : Charles Henri, le compagnon de beuveries et de jeux sexuels, est associé à « un cygne aveugle » qui se distingue par «l'appel pressé du traquet pâtre, le grincement de son bec, et ses coups de sifflet sourcilleux » (Pirotte, 1989 : 41). Sarah devient, à son tour, une linotte mélodieuse, une espèce de passereaux bruns dont le gazouillis est agréable et doux, le vol vif et léger. Pourtant, on retrouve l'emploi péjoratif de ce nom d'oiseau dans le langage des humains. L'expression «tête de linotte » désigne une personne écervelée, agissant étourdiment et à la légère, comme cette espèce d'oiseau qui construit souvent son nid avec insouciance, sans se préoccuper de le dissimuler aux yeux des prédateurs entraînant dès lors la destruction de la nichée. La comparaison est révélatrice. Sarah écrit à propos de sa jeunesse : «Moi-même, je me suis laissé vendre, laissé acheter » (Pirotte, 1989 : 53). Cette phrase souligne le caractère dévastateur de la mémoire, des souvenirs qui condamnent aux remords et qui ne cessent de bouleverser la narratrice.

\footnotetext{
${ }^{1}$ Ce film, intitulé Orphée, est sorti sur les écrans en 1950.

2 À ce propos, voir : M. Augé, Les Formes de l'oubli, Paris, Éditions Payot \& Rivages, 2001.
} 
La fascination de Pirotte pour la figure d'Orphée, dont la musique charmait les animaux sauvages, est également lisible dans la structure du roman qui imite la forme binaire d'une sonate, développée principalement par les compositeurs de tradition germanique. Le premier chapitre du roman correspond à une exposition ; les deux chapitres suivants englobent le travail inspiré par les thèmes de l'exposition avec de nombreuses modalités ; le dernier chapitre n'est qu'une réexposition des thèmes du premier chapitre avec une cadence conclusive. Cette forme binaire de la sonate reflète donc le double mouvement du récit: celui de l'écriture de la mémoire et de l'oubli. Si Orphée parvient à émouvoir les êtres inanimés par les accents de sa lyre, Sarah, cette "feuille morte », est perturbée par un "petit sanglot nauséeux » (Pirotte, 1989 : 123) dans le dernier chapitre de ses écrits sous la forme d'un boucle qui est aussi celle de la sonate.

Dans la quête des souvenirs, ce sont les paysages musicaux, notamment ceux des grands auteurs de sonate - comme Scarlatti ou Mozart - qui déclenchent souvent la mémoire bancale de Sarah. Et la narratrice l'explique ainsi : « la musique connaissait le chemin de ma mémoire, ou ma mémoire celui de la musique » (Pirotte, 1989 : 126). C'est donc l'écho des dernières notes d'une sonatine qui éveille chez Sarah le souvenir de phrases de Sénèque et de Marc Aurèle, citées par son cher ami Carabas. Ces bribes d'aphorismes stoïciens, qui émergent de la mémoire au rythme de la musique, ne sont pas hasardeux. Ils définissent l'homme comme une minime parcelle de la durée totale à qui on n'assigne qu'un infime intervalle. Le mot «intervalle » est l'un des mots-clés du roman pirotien. Selon le calendrier chinois, on entre à partir du 20 octobre dans une intersaison. Le jaunissement des feuilles, qui tombent des arbres, est la transformation majeure qui se produit entre l'automne et l'hiver. Sarah, le double féminin de l'auteur, qui a vu le jour le 20 octobre, est justement née dans cette saison intermédiaire. L'automne devient ainsi, pour la narratrice, la métaphore de l'existence humaine ; elle avoue : "l'automne c'est comme un pays. Un pays pour naître, ce qui est bien vain, mais aussi pour mourir. La vie n'est qu'un interminable automne. Avec le chatoiement des feuilles et le scintillement des grésils, la transhumance des brouillards et l'histoire compliquée des pluies, et ces douceurs passagères qui soudain vous ravissent à vous-même " (Pirotte, $1989: 40$ ).

Or, dans son œuvre, Une ethnologie de soi. Le temps sans âge, Marc Augé recourt à l'expression « hors d'âge », utilisée pour les vieux armagnacs, en constatant :

Un individu "hors d'âge" rassemble plusieurs passés inégalement présents dans sa mémoire, passés recomposés dont souvent les plus anciens ne sont pas les moins tenaces et peuvent lui donner l'impression que sa vie a duré le temps d'un éclair, alors que d'autres, plus récents, mais déjà en voie d'effacement, le persuaderaient aisément d'avoir vécu une éternité, et que d'autres encore flottent dans une brume indistincte à l'horizon de sa mémoire sans qu'il soit en mesure de les situer ou de les dater précisément (Augé, $2014: 45)$. 
Il en est de même avec Sarah, qui note sur la première page de son cahier : « Je suis jeune, je suis vieille, en réalité je n'ai pas d'âge » (Pirotte, 1989: 9). Elle se plonge donc dans des phrases enchevêtrées où elle se contente de laisser venir une « infinité d'images frémissantes, qui compos[ent] un univers instable, inquiet et merveilleux » (Pirotte, 1989: 17) - sans essayer de les dater, de les situer, de les relier. Le roman semble donc être un éloge des «temps ordinaires », où l'Histoire avec un $\mathrm{H}$ majuscule, cède la place à la toute petite vie humaine. De nombreux conflits et changements politiques d'un siècle écoulé s'effacent devant les paysages pirottiens de l'âme.

Dépourvue de «paysage d'enfance» (Pirotte, 1989: 52), Sarah essaie de l'imaginer. Le paradis prend la forme de l'île de Sumatra, à laquelle la narratrice attribue le lieu de naissance de sa grand-mère, qui lui chantait des berceuses javanaises. Elle est l'unique figure de la mémoire familiale dont Sarah garde le doux souvenir. Le seul objet que Sarah conserve de l'époque de "l'enfance anéantie » (Pirotte, 1989 : 115), c'est justement la tête d'une poupée javanaise, offerte par sa grand-mère. À propos de ce jouet, elle note : «C'était ma seule amie. Je n'éprouvais pas le besoin de la bercer, ni de bercer qui que ce soit, ou quoi que ce soit. Je pense que je me berçais moi-même » (Pirotte, 1989 : 16). Pour Sarah, qui s'identifie à un corps de poupée sans tête (Pirotte, 1989 : 15), la tête d'une poupée javanaise devient un pauvre substitut de l'unité perdue, un symbole de l'enfance heureuse, finie à jamais avec la mort de la grand-mère, avec l'abandon par son père et avec l'entrée dans l'univers de beuveries et de jeux pervers de ses tantes à Reims.

Le nom de l'île de Sumatra, située sur l'équateur, signifie en langue malaise «l'océan ». La quête de ce lieu édénique imaginaire est toutefois constamment perturbée par la mémoire de Sarah qui « ressemble à un voilier qui n'a jamais pris la mer» (Pirotte, 1989: 52). Chez Pirotte, les souvenirs sont façonnés par l'oubli comme les contours du rivage par la mer, mais les poussées de l'océan dépendent aussi des formes du relief sous-marin qui constitue le prolongement du paysage terrestre. Il s'agit de la complicité entre la terre et la mer qui, toutes deux, ont contribué à un long travail d'élimination dont le paysage actuel est le résultat. C'est donc la généalogie du paysage actuel, celui du moi présent, que Sarah essaie d'explorer en se livrant à ce travail de sape de la mémoire.

Associée à une sorte de paysage d'enfance par le biais du "souvenir falsifié » (Yves \& Tadié, 1999: 225) ${ }^{3}$ de Sarah, l'île exotique de Sumatra est également l'un des lieux visités par Arthur Rimbaud, poète devenu un marin errant, dont Sarah découvre les livres dans l'appartement de son amant. De même que l'auteur du Bateau ivre, Sarah, abandonnée par son père, s'adonne à une vie vagabonde, aussi bien réelle qu'imaginaire ${ }^{4}$, à travers les paysages mémoriels des lieux de sa jeunesse

\footnotetext{
${ }^{3}$ Le terme « souvenir falsifié » est employé par Jean Yves et Marc Tadié dans un ouvrage théorique, intitulé Le sens de la mémoire.

${ }^{4}$ Toutefois, le voyage lointain d'Arthur Rimbaud correspond à son engagement militaire dans l'armée coloniale hollandaise. Il y déserte en 1876.
} 
où les glissements des lumières et des ombres, des couleurs tamisées et des mille et un nuances la retiennent davantage que les formes et les volumes. L'écriture de la narratrice est donc essentiellement régie par les lieux : les 4 chapitres du roman sont intitulés : Paris, le Val d'or, Les hauts pays, Strasbourg.

Paris constitue un point de départ dans le dédale de la mémoire tandis que Strasbourg marque la fin des déambulations de la narratrice. Les paysages mémoriels des lieux de la jeunesse de Sarah, décrits notamment dans les chapitres aux noms contradictoires, Le Val d'or et Les hauts pays, créent un pont entre la mémoire personnelle de la narratrice et la mémoire collective. Cela est dû au souvenir des vagabondages dans les anciennes provinces historiques de Bourgogne ou de Franche Comté, ainsi qu'à celui des errances à travers les Pays hauts, regroupant une soixantaine de communes allant de la frontière belgo-luxembourgeoise jusqu'aux environs d'Audun-le-Roman. Ces lieux renvoient au territoire historique, appelé par les chroniqueurs Lotharii regnum (« royaume de Lothaire ») ${ }^{5}$. Comme l'île de Sumatra, où vivent des populations qui se désignent elles-mêmes sous le nom de "peuples de la terre", la trace mnésique de la mémoire collective, celle du royaume lotharingien unissant le monde latin et germanique, symbolise la quête de l'unité perdue de la narratrice qui a une double origine : hollandaise (du côté de la mère) et française (de celui du père).

Sarah, le double féminin de Pirotte, ne mentionne qu'une fois les Belges, et le fait de façon ironique; elle parle des Belges hilares qui photographient ${ }^{6}$ le porche de l'ange souriant, en rêvant « d'une sorte d'attestation certifiée conforme d'éternel ou de bon trésor d'amour" (Pirotte, 1989: 71). Cette petite phrase concernant les Belges nous fournit une très importante piste de recherche par rapport à la problématique de la mémoire collective dans l'œuvre pirottienne. Le porche de l'ange souriant correspond à la statue de l'Ange au Sourire dans la cathédrale de Reims ${ }^{7}$, ville où Sarah a passé son adolescence. Lors de la Grande Guerre, la ville de Reims a été pilonnée pendant quatre ans par les tirs d'artillerie de l'armée allemande. Le 19 septembre 1914, suite à un bombardement, une poutre de l'échafaudage s'effondre et décapite l'ange. Sa tête tombe quatre mètres plus bas et se brise en une vingtaine de

\footnotetext{
${ }^{5}$ Jean-Claude Pirotte est l'auteur d'un guide littéraire, consacré justement à la Lotharingie. Ce petit livre de 60 pages fait partie de la collection réalisée pour National Geographic. Voir: J-C. Pirotte, Un rêve en Lotharingie, Tolède, Éditions Pileas Fogg, 2002. Pour plus de détails concernant la fascination de l'auteur pour le royaume de Lothaire, consulter: "L'Europe des connivences : une pensée géopolitique chez Jean-Claude Pirotte ? », un entretien de Hugues Robaye avec Jean-Claude Pirotte, in Confluences, mythes \& histoire, textes réunis et présentés par M. Quaghebeur et L. Rossion, in Confluências, $\mathrm{n}^{\circ} 21$, septembre 2012, pp. 147-165.

${ }^{6}$ La photographie, cet art de fixité, est méprisée par Sarah dont l'écriture nomade est celle entre les lumières et les ombres de la mémoire et son pendant : l'oubli. Il s'agit donc de l'écriture qui se focalise sur l'intervalle, sur la durée que la photographie est incapable de montrer.

${ }^{7}$ C'est un lieu du baptême de Clovis, roi de tous les Francs, ainsi que de la réconciliation francoallemande, officialisée symboliquement en juillet 1962 par Charles de Gaulle et Konrad Adenauer, sous les voûtes de la cathédrale de Reims.
} 
morceaux. La statue devient alors le symbole de la cathédrale martyre et de la barbarie allemande. Il est difficile de ne pas voir les affinités entre la statue de l'ange décapité et le personnage de Sarah qui se définit comme une poupée sans tête. Dans ce contexte, le destin de Sarah, double de l'auteur belge, dont la naissance en 1939 s'accroche à l'éclatement de la Seconde Guerre mondiale, dépasse la mémoire personnelle pour rejoindre la mémoire collective. L'enfance " anéantie », dont parle Sarah, renvoie au traumatisme de tous les hommes dépourvus de l'Eden de l'enfance à cause des atrocités des guerres, et qui deviennent - comme la narratrice - « un vieil enfant » (Pirotte, 1989 : 45) sans avenir.

Dans ses écrits, Sarah revient à l'époque d'après le traumatisme de la guerre, celle de la recherche de l'oubli, qu'elle a passée à Reims en vagabondant d'une boîte à l'autre, d'un amant à l'autre, en s'adonnant à l'ivresse « pour les morts et les blessés » (Pirotte, 1989 : 98). Mais toutes ces activités n'étaient qu'une illusion de l'oubli, car «l'évocation du sang, du beurre noir et du verre brisé transfigurait la tendre Juanita » (Pirotte, 1989: 46) et d'autres compagnons de Sarah, tandis que l'alcool de mirabelle avait « le goût pervers des cadavres frais » (Pirotte, 1989 : 33). Il s'agit donc des années perdues, passées à tisser dans la journée le fil d'oubli qui se défaisait la nuit.

La même cathédrale de Reims, où se trouve la statue de l'Ange au Sourire, cachait autrefois, dans le dallage des $3^{\mathrm{e}}$ et $4^{\mathrm{e}}$ travées de la nef, Le Labyrinthe qui symbolisait la montée du Christ au Calvaire ${ }^{8}$. Cet indice nous mène au dernier chapitre $\mathrm{du}$ roman, où Sarah se souvient d'un ouvrage de Georges Rouault ${ }^{9}$, intitulé Miserere. Il s'agit d'un cycle de gravures, conçu dès 1913. Les évènements de la Grande Guerre mettent en exergue les préoccupations du peintre, qui place le Christ et la mort au premier plan de la scène de Miserere. Édité en 1948, le livre est d'autant mieux compris qu'il succède aux horreurs de la Seconde Guerre mondiale. Ces subtils renvois pirotiens nous permettent d'associer Le Labyrinthe de la Cathédrale de Reims, symbolisant la montée christique au Calvaire, au chemin pénible de Sarah qui, face au traumatisme de la guerre, se perd dans « un dédale d'une enfance disparate » (Pirotte, 1989 : 17). Toutefois, en découvrant dans l'appartement de son amant une aquarelle de Turner $^{10}$, un tableau marin de Pierre Tal Coat ${ }^{11}$, des gravures de Rouault, des livres de Sénèque, de Marc Aurèle et de Rimbaud, ainsi que des disques de Scarletti et de Mozart, qui forment ensemble une partie d'un patrimoine germano-latin, Sarah avoue : « je me trouvais là dans ce décor étranger comme s’il

\footnotetext{
${ }^{8}$ Le Labyrinthe de la Cathédrale de Reims a été choisi comme logo des Monuments historiques.

${ }^{9}$ Georges-Henri Rouault (1871-1958), est un peintre et graveur français.

${ }^{10}$ William Turner (1775-1851), appelé « peintre de la lumière », est le maître de l'aquarelle anglaise

${ }^{11}$ Pierre Tal Coat (1905-1985), est un peintre, graveur et illustrateur français de l'École de Paris. La peinture de Tal Coat s'est développée en séries, notamment: série des Massacres de la guerre d'Espagne, série des Paysages (Bretagne, Bourgogne, Île de France), série des Natures mortes, série des Mouvements d'eau.
} 
m'était familier [...], cela ressemblait à une convalescence après le calvaire d'une amnésie. C'était à la fois trop de mystère et trop d'intimité » (Pirotte, 1989 : 127).

Les morceaux de la tête de l'Ange au Sourire de la Cathédrale de Reims ont été soigneusement ramassés et mis en sécurité dans les caves de l'archevêché à l'époque de la Grande Guerre. En 1926, la tête de la statue, symbolisant la barbarie d'une guerre qui détruisit une partie du patrimoine universel, a été reconstituée à partir des fragments d'origine. Déracinée et dépourvue de "paysage d'enfance » par l'horreur de la guerre, Sarah, elle aussi, a caché les morceaux de sa propre identité dans les profondeurs de la mémoire. Dans sa quête de l'unité perdue, le recours à la mémoire personnelle, contaminée par la mort, s'avère insuffisante. C'est grâce à la reconquête de la mémoire collective, celle d'un patrimoine culturel, unissant culture latine et germanique, que Sarah semble retrouver les fragments de son origine, indispensables à la reconstruction de Soi ${ }^{12}$. Ainsi, annonce-t-elle le destin du héros pirottien du roman Fond de cale, qui avoue : « je ne suis pas né d'une femme, je suis né d'une phrase, d'une métaphore dans un livre, ou d'une ombre de brume sur la mer » (Pirotte, 1991 : 119).

Bien que Pirotte recoure, de façon implicite et habilement codée, à l'Histoire, celle-ci, basée sur les principes de logique, de causalité et de raison, se fond dans le kaléidoscope des paysages mémoriels des sons, des territoires naturels, urbains ou picturaux, des phrases propres et empruntées aux écrivains, car « la peinture, la musique, les oiseaux, la littérature même, c'est une énigme éblouissante, $[\ldots]$ nous sommes au cœur de mystère. Non pas en face, ni alentour. Au cœur même ", explique le narrateur pirottien de Plis perdus (Pirotte, 1994 : 62).

Sarah, qui par sa double origine - hollandaise et française - appartient à une vaste culture germano-latine, accède finalement à une sorte de jardin édénique ${ }^{13}$, situé dans le centre historique de Strasbourg, classé patrimoine mondial en 1988 et appellé la Grande Ille (ou ellipse insulaire). Cet Eden est celui de la ville cosmopolite, multiculturelle et hétéroclite qui a accueilli, entre autres, Gutenberg et des grands humanistes européens comme Didier, Erasme, Goethe. C'est aussi la ville qui est devenue le symbole de la réconciliation franco-allemande et de l'Union européenne dont l'idée principale était de créer les conditions d'une paix durable en Europe. Ce terminus pirottien unit donc la mémoire personnelle de Sarah, représentée par le

${ }^{12}$ Dans le roman, Absent de Bagdad (2007), Pirotte se confronte de façon explicite à l'Histoire en y exposant sa critique radicale des politiques menées par les pays occidentaux et des sociétés qu'elles installent. Le narrateur, présumé musulman alors qu'il parle turc, kurde, anglais, lit Bernanos et Montaigne, est enfermé dans un cul-de-basse-fosse. Condamné à la souffrance physique et psychique de la part de ses gardiens américains, il découvre la liberté, celle d'aimer l'humanité par-delà de sa barbarie en se souvenant des poèmes, des textes philosophiques ou des paroles des chansons appartenant au patrimoine universel qui rejette les frontières entre l'Europe occidentale et l'Orient. Pour plus de détails concernant ce roman, voir : M. Quaghebeur, «Clairière inespérée ». La mémoire nomade et la dignité : Absent de Bagdad de Jean-Claude Pirotte, à paraître.

${ }^{13}$ À ce propos, voir: S. Lejeune, Paradis perdus et retrouvés dans quelques ouvres de JeanClaude Pirotte, à paraître. 
souvenir falsifié du paysage de l'enfance, celui d'une île exotique de Sumatra et une mémoire collective régie par l'image du royaume de Lothaire et du patrimoine culturel universel.

De plus, les sujets obsédants du roman pirottien, notamment ceux de l'impuissance maladive d'affronter le présent, du culte de l'enfance, du nomadisme de l'esprit, de la recherche de l'identité dans un « pays improbable » (Pirotte, 1989: 88), de la nostalgie de l'unité perdue et de la quête de l'espace idéal, semblent refléter l'état des mentalités dans la Belgique francophone de l'après 1980, et d'avant le $\mathrm{XXI}^{\mathrm{e}}$ siècle. Le choix de Strasbourg, ville au carrefour de deux univers, germanique et latin, comme terminus du voyage de Sarah, est donc tout sauf le fruit du hasard. Mais ce roman, qui voit le jour en 1989, année de la chute du mur de Berlin, dépasse le cadre de la mémoire belge et lotharingienne. Lorsque Sarah se demande à Strasbourg : « Irai-je jamais plus loin vers l'Est » (Pirotte, 1989 : 92), Pirotte suggère la réponse : Strasbourg, dont le nom signifie « le château sur la route ${ }^{14}$, se trouve à égale distance de la Baltique et du littoral atlantique, de la Méditerranée, de la mer du Nord et de l'Adriatique. Il s'agit donc d'un lieu symbolique au carrefour de l'Europe qui rend impossible l'idée de n'importe quel Rideau de fer. Le voyage de Sarah comme celui du Bateau ivre rimbaldien devient donc un passeport vers la voyance.

Après la reconstruction, l'Ange au Sourire de la Cathédrale de Reims semble envisager l'avenir avec sérénité. Sarah, dans le jardin de Strasbourg, semble se libérer d'un poids du passé, qui la retenait dans « un temps immobile » ( $S$, p. 23) et la privait de l'avenir. Elle dit : « Je me sens ici, enfin, très loin de tout, très loin de ma vie, ou plutôt, je devrais dire très près d'une autre vie qui est également indifférente mais miroite ainsi qu'un lac gelé [...] c'est encore à l'enfance que je rêvais et puis la nouvelle de la mort de mon père a réveillé d'autres visions mais cela est bien étouffé maintenant » $(S, \mathrm{p}$. 130). Pour aboutir à ce nouvel état d'esprit, celui de la continuité entre le passé et le présent, il fallait pourtant se livrer à l'écriture de la mémoire qui fait un lien entre la succession des moi qui ont existé depuis notre conception jusqu'à l'instant présent. Cette écriture labyrinthique entraîne celle de l'oubli qui est l'adaptation de l'être au présent de l'esprit et qui permet d'éviter l'enfer de certains souvenirs en les remplaçant par faux souvenirs, ceux à mi-chemin entre le souvenir et l'oubli.

Suite au retour d'Ulysse, qui mit dix ans à revenir dans son île d'Ithaque et y retrouver son épouse, Pénélope peut enfin abandonner son stratagème de tisser la toile le jour pour la défaire la nuit. Sarah, à son tour, finit son écriture pénélopéenne, celle de la mémoire et de l'oubli, après avoir retrouvé sa propre Ithaque sur la Grande Île de Strasbourg, ville où se trouve l'ancien cinéma, L'Odyssée $e^{15}$, inscrit aux monuments historiques.

\footnotetext{
${ }^{14}$ Sarah et son ami Pierrot rêvent d'une vie fabuleuse dans un château (Pirotte, 1989 : 98).

${ }^{15}$ Construit en 1913, et inscrit aux monuments historiques, L'Odysée est l'un des plus anciens cinémas de France.
} 


\section{BIBLIOGRAPHIE}

Augé, M. (2014). Une ethnologie de soi. Le temps sans âge. Paris : Seuil.

"L'Europe des connivences : une pensée géopolitique chez Jean-Claude Pirotte ? », un entretien de Hugues Robaye avec Jean-Claude Pirotte in Confluences, mythes \& histoire, textes réunis et présentés par M. Quaghebeur et L. Rossion, Confluências, 21, septembre 2012, 147-165.

Pirotte, J.-C. (1989). Sarah, feuille morte. Cognac : Le temps qu'il fait.

- (1991). Fond de cale. Cognac : Le temps qu'il fait.

- (1994). Plis perdus. Paris : La Table Ronde.

- (2002). Un rêve en Lotharingie, Tolède : Éditions Pileas Fogg.

Yves, J., Tadié, M. (1999). Le sens de la mémoire. Paris : Gallimard. 